\title{
Performance Evaluation of Base-Station-Assisted Link State Routing Method for Mobile Ad Hoc Networks
}

\author{
Hiroaki Morino $^{1}$, Tadao Saito ${ }^{1,2}$, and Mitsuo Nohara ${ }^{2}$ \\ 1 Research and Development Initiative Chuo University \\ 1-13-27 Kasuga Bunkyo-ku Tokyo 112-8551, Japan \\ Tel: +81-3-3817-1626 Fax: +81-3-3817-1606 \\ morino@m.ieice.org \\ 2 TOYOTA InfoTechnology Center Co., Ltd.
}

\begin{abstract}
For communication systems of automobiles, hot spot networks using wireless LAN are attractive in that broad bandwidth is available at low cost. Further, inter-vehicle ad hoc networks organized by cars are expected to cooperate with the hot spot network and effectively extend the coverage of the hot spot network. The authors have proposed base-station-assisted link state routing method(BALSR) 1 that is effective for large scale inter-vehicle ad hoc networks. This paper presents performance evaluation of BALSR, and it shows that BALSR significantly reduces the number of conrol packets in the ad hoc network compared to conventional link state routing method.
\end{abstract}

Keywords: Inter-vehicle mobile ad hoc network, link state routing method, wireless LAN base station

\section{Introduction}

Recently, broadband wireless communication systems including 3G cellular system, hot spot networks by wireless LAN and so on have emerged, and capacity of communication systems of automobiles is increasing from tens of kilo bit/sec to hundreds of kilo bit/sec. Especially, hot spot networks are attractive for the availability of broad bandwidth at the lower cost. Although coverage of hot spot networks is limited, it will be effective when some terminals in the coverage receive data from the hot spot network and they share received data with other terminals out of the coverage using the inter-vehicle ad hoc network. This is called the information delivery system in this paper. In this system, performance of throughput will be largely affected by the routing method of inter-vehicle ad hoc network. For the routing method, mainly the following three conditions will be required; (1) Many communication pairs are handled efficiently (2) The method works with low control overhead in the case that the ad hoc network is large scale whose size is several kilometers (3) The route between the information sender terminal to the information receiver terminal can be constructed in a few seconds in order that the system efficiently works. 
There have been many proposals of routing methods for mobile ad hoc networks. For example, MANET(Mobile Ad-hoc NETworks) Working Group of IETF (Internet Engineering Task Force) discusses mainly two types of routing methods. One is the proactive method, where every terminal maintains routing table by periodic exchange of routing information, and OLSR $[2$ and TBRPF 3 ] are examples of this method. The other is the reactive method, where only terminals that have data to send construct routes on demand. AODV 4 and DSR 5] are examples of this method. In these methods, however, each terminal has to relay packets of routing information more frequently as the number of hops of the network gets large. In the wireless LAN based ad hoc network, it is possible that excessive amount of control packets causes congestion that leads to throughput decrease of the network. From this viewpoint, we have proposed base-station-assisted link state routing method (BALSR).

This paper presents performance evaluation of BALSR, and it shows that BALSR reduces the number of control packets in ad hoc mode wireless LAN in inter-vehicle communications.

\section{Overview of BALSR}

\subsection{Overview of the Information Delivery Systems Using Hot Spot Network and Inter-vehicle Communication}

Figure 1 illustrates overview of the information delivery system using the hot spot network and the inter-vehicle ad hoc network. In this figure, the car (a), which is out of coverage of the hot spot network, wants to receive some data from the network. It is called information receiver terminal. Car (b) is one of terminals newly coming into the coverage of the hot spot network, and it is called information sender terminal. The procedures of the system for information delivery between these two terminals are as follows.

(1) The information sender terminal begins to join the inter-vehicle ad hoc network and constructs routes to other all of terminals that already joined the network.

(2) When the route between the information receiver terminal and the information sender terminal is established, the information receiver terminal sends request to the information sender terminal for receiving data from the hot spot network.

(3) The information sender terminal receiver the requested data from the hot spot network and sends the data to the information receiver terminal.

\subsection{Operations of BALSR}

Figure 2 shows an overview of the inter-vehicle ad hoc network realized by BALSR. In this system, wireless LAN base stations are provided along the road for routing control of the network. Each terminal has two wireless LAN interfaces; one is ad hoc mode wireless LAN for inter-vehicle communication and 


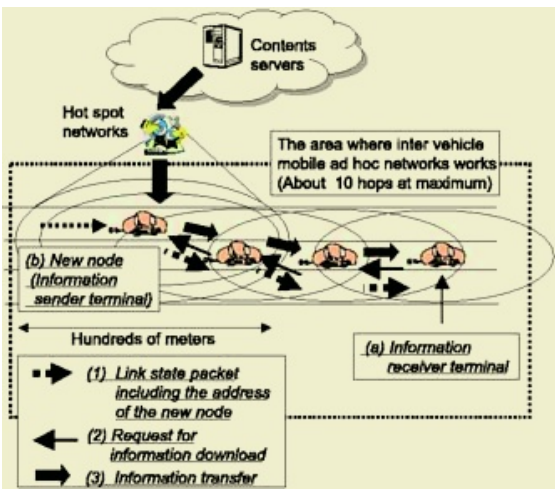

Fig. 1. Information delivery system us- Fig. 2. Overview of the base-station-assisted

ing the hot spot network and inter- link state routing method vehicle mobile ad hoc networks

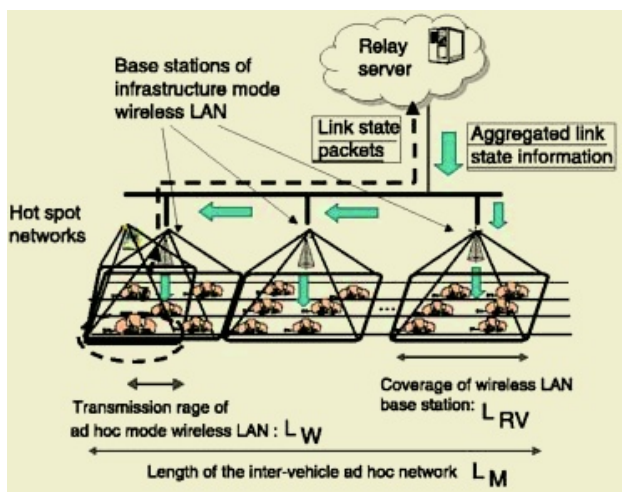

another is infrastructure mode wireless LAN to communicate with base stations. Infrastructure mode wireless LAN is configured so that it has larger transmission range and lower bit rate compared to ad hoc mode wireless LAN. Functions of wireless LAN base stations described here can be also realized by base stations of cellular system in the case that the cellular system supports point-to-multipoint communications. Operations of the system are as follows. Each terminal sends the hello packet in the certain interval $\mathrm{T}$ (hello) by ad hoc mode wireless LAN and recognizes neighbor terminals. Then the terminal generates the link state packet in the certain interval $\mathrm{T}$ (ls) that includes link status information between itself and neighbor terminal, and it sends it to the relay server via wireless LAN base stations. The relay server receives link state packets sent by terminals, aggregates link state information in these packets, and sends the information to all terminals via wireless LAN base stations to all terminals at the certain period. The period at which the relay server sends link state information to all terminals is called packet relay period, and it is denoted by $\mathrm{T}$ (relay).

In this method, the number of control packets handled in ad hoc mode wireless LAN is significantly reduced, and throughput will be improved.

\section{Performance Evaluation}

\subsection{Evaluation Model}

\section{The Model of Car Movement and Data Packets Transmission between}

Cars. The model of car movement is shown in Fig.3, where each car runs at a constant speed and keeps a constant distance to a car ahead. The model of data packets transmission between cars is as follows. Among cars that newly enter the area of the ad hoc network, predetermined numbers of cars are selected as information sender terminals. For each of these terminals, a car most distant from it is selected as an information receiver terminal, and the information sender 
terminal sends data packets to the information receiver terminal by unicast for 20 second, at a sending rate of 10 packet/sec. When 20 seconds have passed, the information sender terminal selects another information receiver terminal and repeats the same procedure until it reaches at the end of the area of the network.

Parameters of Wireless LAN and Routing Method. In the model of BALSR shown in Fig.2, parameters of wireless LAN are as follows.

- Ad hoc mode wireless LAN

- Bit rate : $2 \mathrm{Mbps}$

- Transmission range $L_{W}: 150-300 \mathrm{~m}$

- Infrastructure mode wireless LAN

- Bit rate : $128 \mathrm{kbps}$

- Coverage of base station $L_{R V}: 1 \mathrm{~km}$

Parameters of ad hoc mode wireless LAN are determined to model IEEE 802.11b 2Mbps mode. On the other hand, parameters of infrastructure mode wireless LAN are determined by assuming that the system has larger spreading factor than IEEE 802.111b. In the ad hoc mode wireless LAN, packets sent from a terminal arrive at the next terminal when the distance between two terminals is equal or less than $L_{W}$. It is assumed that packets are lost when distance is larger than $L_{W}$, and lost packets are not retransmitted. It is also assumed that any packet loss caused by collision does not occur Routing methods evaluated are BALSR and OLSR. In both methods, interval of hello packets T(hello) is 2 seconds. Interval of link state packets $\mathrm{T}(\mathrm{ls})$ is 2 seconds at minimum and 6 seconds at maximum respectively. Packet relay period T(relay) in BALSR is set as 100msec. In BALSR and OLSR, parameters are configured so that routes between terminals within two hops are constructed in mesh topology, and routes between terminals distant by more than two hops are constructed in tree topology.

At last, the way of utilization of wireless LAN base stations in BALSR and OLSR is as follows. Though wireless LAN base stations are provided mainly for control of the ad hoc network, it is assumed that they are connected to content servers shown in Fig.1 and each terminal in both method receive information from content servers via them.

\subsection{Results}

Relationship between Data Packet Delivery Ratio and Transmission Range. Figure 4 shows data packets delivery ratio in BALSR. Data packets delivery ratio is defined as the ratio of data packets that arrived at the information receiver terminal among data packets sent from information sender terminal. Since BALSR and OLSR has almost the same data characteristics, the figure only shows results of BALSR. The figure shows that data packet delivery ratio in the network with the same value of maximum number of hops(calculated by 


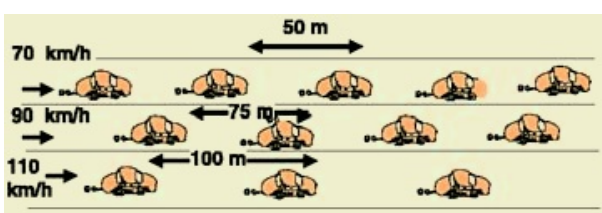

Fig. 3. Car movement model in the sytem Fig. 4. Data packet delivery ratio in shown in Fig.2

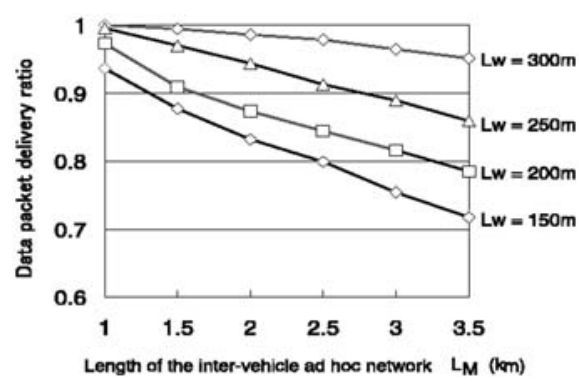

BALSR

$\left.L_{M} / L_{W}\right)$ is improved as $L_{W}$ increases. This is by the effect that routes between terminals within two hops are configured to be constructed in mesh topology, as described in Section 3.1 If a route fails between some two terminals within two hops by topology change, an alternative route can be immediately established among redundant routes already established in mesh topology. From this results, it can be said that $L_{W}$ should be set as large value as possible to improve data packet delivery ratio in large scale network.

Control Overhead. Figure 5 shows the number of conrol packets that ad hoc mode wireless LAN in each terminal and each wireless LAN base station sends and receives in BALSR and OLSR. In OLSR, it increases as $L_{M}$ increases, and it reaches 76 packets $/ \mathrm{sec}$ when $L_{M}=3 \mathrm{~km}$. On the other hand, BALSR reduces the number of control packets in each terminal to 22 packets/sec, which is about $30 \%$ of that of OLSR. Fig. 6 shows amount of information of control packets each terminal and each wireless LAN base station sends and receives in BALSR and OLSR. BALSR reduces amount of information of control packets handled in each terminal by about 50kbps compared to OLSR. On the other hand, BALSR consumes about bandwidth of each wireless LAN base station by about 25kbps. Therefore, it can be said that total bandwidth that control packets consume in ad hoc mode wireless LAN and wireless LAN base stations in BALSR is almost the same as that of OLSR.

From these results, it can be concluded that BALSR is effective in that it reduces the number of control packets in ad hoc mode wireless LAN compared to OLSR. It is also shown that total bandwidth consumed by control packets in ad hoc mode wireless LAN and wireless LAN base station in BALSR is almost the same as OLSR.

\section{Conclusion}

This paper presents performance evaluation of BALSR in large scale inter-vehicle ad hoc networks under the conditions that the inter-vehicle ad hoc network works in order to effectively extend the coverage of the hot spot network. 


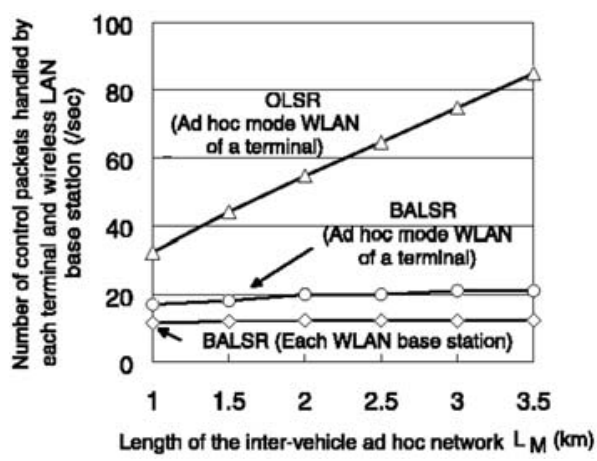

Fig. 5. The number of control packets handled by each terminal and each wireless LAN base station

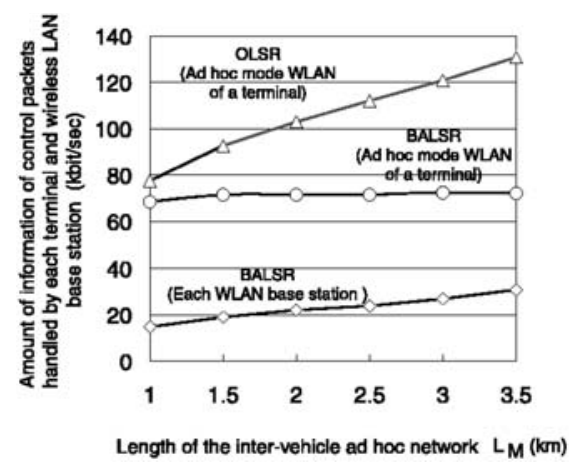

Fig. 6. Amount of information of control packets handled by each terminal and each wireless LAN base station

Firstly, data packet delivery ratio is evaluated under the condition of various transmission ranges of ad hoc mode wireless LAN. It is found that BALSR achieves data packet delivery ratio above $90 \%$ in the case that the length of the ad hoc network is $3 \mathrm{~km}$, when the range is equal to or greater than $300 \mathrm{~m}$. Secondly, the number of control packets that ad hoc mode wireless LAN in each terminal sends and receives is evaluated. It is shown that BALSR reduces the number of control packets to $30 \%$ of that of OLSR in the case that the length of ad hoc network is $3 \mathrm{~km}$. This reduces load of ad hoc mode wireless LAN in each terminal, and it will lead to improve throughput of the ad hoc network. For future work, throughput performance of BALSR will be further evaluated in detail by considering wireless LAN media access control.

Acknowledgements. The authors wish to thank Prof. Shigehiko Naoe (Chuo University), Mr. Tadao Mitsuda (TOYOTA InfoTechnology Center Co., Ltd) and Prof. Takeshi Hattori (Sophia University) for their valuable comments.

\section{References}

1. H. Morino et al., "An efficient proactive routing method for mobile ad hoc networks using peer-to-peer and cellular communication system," PWC 2002 Oct 2002.

2. Phillipe Jacquet et al., "Optimized Link State Routing Protocol," Proc. of IEEE INMIC 2001.

3. Richard Ogier et al., "Topology Dissemination Based on Reverse-Path Forwarding," IETF Internet-Draft < draft-ietf-manet-tbrpf-07.txt> Mar 2003.

4. C. Perkins et al., "Ad hoc On-demand Distance Vector (AODV) Routing," Internet-Draft < draft-ietf-manet-aodv-10.txt> Jan 2002.

5. David B. Johnson et al., "The Dynamic Source Routing Protocol for Mobile Ad Hoc Networks (DSR)", Internet-Draft < draft-ietf-manet-dsr-07.txt> Feb 2002. 